MATHEMATICS OF COMPUTATION

Volume 81, Number 278, April 2012, Pages 905-922

S 0025-5718(2011)02518-4

Article electronically published on July 12, 2011

\title{
MATRICIAL FILTERS AND CRYSTALLOGRAPHIC COMPOSITE DILATION WAVELETS
}

\author{
JEFFREY D. BLANCHARD AND ILYA A. KRISHTAL
}

\begin{abstract}
In 2006 Guo, Labate, Lim, Weiss, and Wilson introduced the theory of MRA composite dilation wavelets. We continue their work by studying the filter properties of such wavelets and present several important examples.
\end{abstract}

\section{INTRODUCTION}

Composite dilation wavelets $(\mathrm{CDW})$ represent a relatively recent [9, 10] and rapidly developing direction in the study of reproducing systems of the Hilbert space $L^{2}\left(\mathbb{R}^{n}\right)$. CDW differ from the standard wavelets because they generate bases (or frames) using two groups of dilations. One group is associated with an expanding matrix $a \in G L_{n}(\mathbb{R}),|\operatorname{det}(a)|=L+1$, (just like the standard wavelets), and the other with a group $B \subset \widetilde{S L_{n}}(\mathbb{R})$ of matrices $b \in B$ with $|\operatorname{det}(b)|=1$. Both types of wavelets use translation operators associated with a lattice $\Gamma=c \mathbb{Z}^{n}$ for some $c \in G L_{n}(\mathbb{R})$. In a composite wavelet, the dilations from $B$ and the translations from $\Gamma$ act together as "shifts" while $a$ continues to perform expanding dilations.

The most widely used example of the group $B$ is the shear group,

$$
B=\left\{b=\left(\begin{array}{ll}
1 & j \\
0 & 1
\end{array}\right) ; j \in \mathbb{Z}\right\},
$$

that gives rise to shearlets [6. While shearlets have found good success in certain applications (e.g. [5, 7, 8), R. Houska showed in his thesis 12 that it is impossible to find orthogonal, MRA shearlets with compact support in the time domain. Recently Kutyniok, Lemvig, and Lim have developed non-MRA compactly supported shearlet frames [14, 15. However, it is possible to find orthogonal, compactly supported, MRA CDW in the case of a finite group $B$ (e.g. [3, 13]). The goal of this paper is to develop a theoretical, matricial filter base for such examples and present some new compactly supported CDW.

Throughout the entire article we assume that the group $B$ is finite and that it is normalized by the dilation $a$, that is, $a^{-1} B a=B$. Another important assumption is the crystallographic condition (or restriction) which requires the group $B$ to fix the full rank lattice $\Gamma$, i.e. $B(\Gamma)=\Gamma$. When the group $B$ and lattice $\Gamma$ satisfy the crystallographic condition, we say that $B$ (or more formally the semi-direct product

Received by the editor November 22, 2009 and in revised form, January 16, 2011.

2010 Mathematics Subject Classification. Primary 42C40.

Key words and phrases. Filters, wavelets, composite dilation wavelets, bracket product, unitary extension principle.

The first author was partially supported by NSF DMS (VIGRE) Grant number 0602219.

The second author was partially supported by NSF DMS Grant number 0908239. 
$B \ltimes \Gamma)$ is a crystallographic group. The normalization and crystallographic conditions play a fundamental role in our analysis because they allow us to treat CDW as a special case of ordinary multiwavelets with some additional composite symmetry. Because of this, we choose to first present the matrix filter theory for general multiwavelets and then concentrate on the study of the composite symmetry.

The paper is organized as follows. The next section introduces the necessary notation, the definitions of composite MRA and CDW, and presents a few technical "toolbox" results that are more or less standard for an MRA theory. In Section

3 we develop basic matrix filter theory for multiwavelet MRA and study in that language the composite symmetry that turns ordinary multiwavelets into CDW. Finally, in Section 4 we present three different examples of CDW illustrating the theory of Section 3 .

\section{BASIC DEFINITIONS AND TOOLBOX RESUlTS}

We begin with the definitions of multigenerated wavelets and CDW. As usual, $n \in \mathbb{Z}, \Gamma=c \mathbb{Z}^{n}$ for $c \in G L_{n}(\mathbb{R})$, and $a \in G L_{n}(\mathbb{R})$ is an expanding matrix with $|\operatorname{det}(a)|=L+1$. Again, as usual, dilations and translations are unitary operators on $L^{2}\left(\mathbb{R}^{n}\right)$ defined by

$$
\left(D_{a} f\right)(x)=|\operatorname{det}(a)|^{-1 / 2} f\left(a^{-1} x\right), \quad\left(T_{k} f\right)(x)=f(x-k), k \in \Gamma, f \in L^{2}\left(\mathbb{R}^{n}\right) .
$$

Definition 1 (Multiwavelet). $\Psi=\left(\psi^{1}, \ldots, \psi^{L}\right) \subset L^{2}\left(\mathbb{R}^{n}\right)$ is an (orthonormal) multiwavelet if there exists an expanding matrix $a$ and a full rank lattice $\Gamma$ such that the affine system $\mathcal{A}_{a \Gamma}(\Psi)=\left\{D_{a}^{j} T_{k} \psi^{\ell}: j \in \mathbb{Z}, k \in \Gamma, 1 \leq \ell \leq L\right\}$ is an orthonormal basis of $L^{2}\left(\mathbb{R}^{n}\right)$.

To define a CDW we use an $(m+1)$-element subgroup $B=\left\{b_{0}=I_{n}, b_{1}, \ldots, b_{m}\right\}$ of $G L_{n}(\mathbb{R})$ and unitary dilations

$$
f_{s}(x)=D_{b_{s}} f(x)=f\left(b_{s}^{-1} x\right), \quad s=0,1, \ldots, m, f \in L^{2}\left(\mathbb{R}^{n}\right) .
$$

Definition 2 (Composite Dilation Wavelet). $\Psi=\left(\psi^{1}, \ldots, \psi^{L}\right) \subset L^{2}\left(\mathbb{R}^{n}\right)$ is an (orthonormal) composite dilation wavelet if there exists an expanding matrix $a$, a group of invertible matrices $B$, and a full rank lattice $\Gamma$ such that the composite dilation wavelet system $\mathcal{A}_{a B \Gamma}(\Psi)=\left\{D_{a}^{j} D_{b} T_{k} \psi^{\ell}: j \in \mathbb{Z}, b \in B, k \in \Gamma, 1 \leq \ell \leq L\right\}$ is an orthonormal basis of $L^{2}\left(\mathbb{R}^{n}\right)$.

The crystallographic condition $B(\Gamma)=\Gamma$, and the commutation relationship $D_{b} T_{k}=T_{b k} D_{b}$ ensure that the system $\mathcal{A}_{a B \Gamma}(\Psi)$ is, indeed, a special case of the system $\mathcal{A}_{a \Gamma}(\Psi)$. More precisely, $\mathcal{A}_{a B \Gamma}(\Psi)=\mathcal{A}_{a \Gamma}(\tilde{\Psi})$, where $\tilde{\Psi}=\left(D_{b} \psi^{\ell}\right), b \in B$, $1 \leq \ell \leq L$.

Definition 3 (Multigenerated MRA). A nested sequence, $\left\{V_{j}\right\}_{j \in \mathbb{Z}}$, of closed subspaces of $L^{2}\left(\mathbb{R}^{n}\right)$ is a multigenerated $(a, \Gamma)$-multiresolution analysis (MRA) if all of the following conditions are satisfied:

(M1) $V_{j} \subset V_{j+1}$ where $V_{j}=D_{a}^{-j} V_{0}$

(M2) $\bigcup_{j \in \mathbb{Z}} V_{j}=L^{2}\left(\mathbb{R}^{n}\right)$;

(M3) $\bigcap_{j \in \mathbb{Z}} V_{j}=\{0\}$;

(M4) there exist scaling functions $\varphi_{0}, \varphi_{1}, \ldots, \varphi_{m} \in V_{0}$ such that $\left\{T_{k} \varphi_{s}: 0 \leq s \leq\right.$ $m, k \in \Gamma\}$ is an orthonormal basis for $V_{0}$. 
As in the classical case condition (M3) is not independent; it follows from the rest of the conditions in the MRA [11].

In this paper we are most interested in the case when the scaling functions $\varphi_{0}, \varphi_{1}, \ldots, \varphi_{m}$ satisfy the additional symmetry constraint defined by (2.1). Let $\varphi \in L^{2}\left(\mathbb{R}^{n}\right)$ and define

$$
\varphi_{s}(x)=D_{b_{s}} \varphi(x)=\varphi\left(b_{s}^{-1} x\right), \quad s=0,1, \ldots, m .
$$

When $\varphi=\varphi_{0}, \ldots, \varphi_{m}$ satisfy (M4), we call $\varphi \in L^{2}\left(\mathbb{R}^{n}\right)$ a composite scaling function. Using the crystallographic restriction $B(\Gamma)=\Gamma$ we see that a special case of a multigenerated MRA is the composite MRA defined as follows.

Definition 4 (Composite MRA). A nested sequence, $\left\{V_{j}\right\}_{j \in \mathbb{Z}}$, of closed subspaces of $L^{2}\left(\mathbb{R}^{n}\right)$ is an $(a, B, \Gamma)$-multiresolution analysis if all of the following conditions are satisfied:

(M1) $V_{j} \subset V_{j+1}$ where $V_{j}=D_{a}^{-j} V_{0}$;

(M2) $\overline{\bigcup_{j \in \mathbb{Z}} V_{j}}=L^{2}\left(\mathbb{R}^{n}\right)$;

(M3) $\bigcap_{j \in \mathbb{Z}} V_{j}=\{0\}$;

(M4) there exists $\varphi \in V_{0}$ such that $\left\{D_{b} T_{k} \varphi: b \in B, k \in \Gamma\right\}$ is an orthonormal basis for $V_{0}$.

As usual, we say that a multiwavelet is associated with a multigenerated MRA $\left\{V_{j}\right\}_{j \in \mathbb{Z}}$ if all of its components belong to the space $W_{0}=V_{1} \ominus V_{0}$. It is easily shown (see, e.g., 10 for a similar argument) that in this case the multiwavelet has exactly $L(m+1)$ components: $\Psi=\left(\psi_{s}^{\ell}\right), 1 \leq \ell \leq L=|\operatorname{det}(a)|-1,0 \leq s \leq m$.

It is convenient to use vector function notation to express multiscaling functions and multiwavelets. We let $F: \mathbb{R}^{n} \rightarrow \mathbb{R}^{m+1}$ be the column vector-valued function

$$
F(x)=\left[\begin{array}{c}
f_{0}(x) \\
\vdots \\
f_{m}(x)
\end{array}\right] \in\left(L^{2}\left(\mathbb{R}^{n}\right)\right)^{m+1} .
$$

In particular, a multiscaling function of a multigenerated MRA is the vector function $\Phi=\left(\varphi_{0}, \ldots, \varphi_{m}\right)^{t}$. If the symmetry property (2.2) is satisfied, then $\Phi$ is called a multiscaling function of the composite MRA. Similarly, we let $\Psi^{\ell}=\left(\psi_{0}^{\ell}, \ldots, \psi_{m}^{\ell}\right)^{t}$, $1 \leq \ell \leq L$. For the sake of brevity, we define $\Psi^{0}=\Phi$ and interchange the notation if we want to emphasize the scaling function.

We also make use of the following shift-invariant subspaces of $L^{2}\left(\mathbb{R}^{n}\right)$ :

$$
\langle F\rangle_{\Gamma}=\left\langle f_{s}\right\rangle_{\Gamma}:=\overline{\operatorname{span}}\left\{T_{k} f_{s}: k \in \Gamma, 0 \leq s \leq m\right\}
$$

and

$$
\langle\langle f\rangle\rangle_{B \Gamma}:=\overline{\operatorname{span}}\left\{D_{b} T_{k} f: b \in B, k \in \Gamma\right\} .
$$

By the crystallographic condition, $\langle\langle f\rangle\rangle_{B \Gamma}=\left\langle f_{s}\right\rangle_{\Gamma}$ if the functions $f_{s}$ satisfy (2.1).

We use the Fourier transform in the form

$$
\hat{f}(\xi)=\int_{\mathbb{R}^{n}} f(x) e^{-2 \pi i \xi x} d x .
$$

It is well defined by the standard extension to $L^{2}$ and because we adopt the convention that elements of the Fourier (frequency) domain are row vectors while the elements of the spatial (time) domain are column vectors. The Fourier transform of a vector-valued function defined by (2.3) is taken componentwise. 
In the following analysis, we employ the bracket product notation generalized to the full rank lattice $\Gamma=c \mathbb{Z}^{n}$ for some $c \in G L_{n}(\mathbb{R})$. The dual lattice to $\Gamma$ is the full rank lattice $\Gamma^{*}=\hat{\mathbb{Z}}^{n} c^{-1}$, where $\hat{\mathbb{Z}}^{n}$ denotes the set of integer row vectors and not the Pontryagin dual of the group $\mathbb{Z}^{n}$. Of course, if $k \in \Gamma$ and $\gamma \in \Gamma^{*}$, then $\gamma k \in \mathbb{Z}$. By $\mathbb{T}_{\Gamma}$ we shall denote the quotient group $\mathbb{R}^{n} / \Gamma$.

Definition 5 (Bracket Product). Given a lattice $\Gamma=c \mathbb{Z}^{n}$ for some $c \in G L_{n}(\mathbb{R})$, the $\Gamma$-bracket product of $f, g \in L^{2}\left(\mathbb{R}^{n}\right)$ is defined by

$$
[f, g]_{\Gamma}(x)=|\operatorname{det}(c)| \sum_{k \in \Gamma} f(x+k) \overline{g(x+k)} .
$$

It follows from (2.5) that $[\hat{f}, \hat{g}]_{\Gamma^{*}}(\xi)=|\operatorname{det}(c)|^{-1} \sum_{\gamma \in \Gamma^{*}} \hat{f}(\xi+\gamma) \overline{\hat{g}(\xi+\gamma)}$. We also use the bracket product notation for vector functions.

Definition 6 (Vector Bracket Product). Given a lattice $\Gamma=c \mathbb{Z}^{n}$ for some $c \in$ $G L_{n}(\mathbb{R})$, the $\Gamma$-bracket product of $F, G \in\left(L^{2}\left(\mathbb{R}^{n}\right)\right)^{m+1}$ is an $(m+1) \times(m+1)$ matrix defined by

$$
[F, G]_{\Gamma}(x)=|\operatorname{det}(c)| \sum_{k \in \Gamma} F(x+k) G^{*}(x+k)=\left[\left[f_{s}, g_{s^{\prime}}\right]_{\Gamma}(x)\right]_{s, s^{\prime}=0}^{m+1} .
$$

The $\Gamma$-bracket product is, clearly, a sesquilinear form. Its other important properties are collected in the following lemma, different versions of which appear throughout the literature for $\mathbb{Z}^{n}$-shift-invariant spaces. We cite [1, Proposition 2.10] as an example.

Lemma 1. Let $f, g \in L^{2}\left(\mathbb{R}^{n}\right), F, G \in\left(L^{2}\left(\mathbb{R}^{n}\right)\right)^{m+1}$ be non-zero vector functions, $\Gamma=c \mathbb{Z}^{n}$ for $c \in G L_{n}(\mathbb{R})$, and $\mathbb{T}_{\Gamma}=\mathbb{R}^{n} / \Gamma$. Then

(i) The series (2.5) converges absolutely a.e. to a function in $L^{1}\left(\mathbb{T}_{\Gamma}\right)$.

(ii) For $k \in \Gamma,\left\langle T_{k} f, g\right\rangle$ is the $k$-th Fourier coefficient of the function $[\hat{f}, \hat{g}]_{\Gamma^{*}}$.

(iii) $\langle F\rangle_{\Gamma} \perp\langle G\rangle_{\Gamma}$ if and only if $[\hat{F}, \hat{G}]_{\Gamma^{*}}=0$ a.e.

(iv) $F$ generates an o.n.b. for $\langle F\rangle_{\Gamma}$ if and only if $[\hat{F}, \hat{F}]_{\Gamma^{*}}=I_{m+1}$ a.e.

(v) $[M F, N G]_{\Gamma}(x)=M(x)[F, G]_{\Gamma}(x) N^{*}(x)$ if $M, N$ are $\Gamma$-periodic $(m+1) \times$ $(m+1)$ matrices.

Proof. We omit (i), while (iii) and (iv) easily follow from (ii), which, in turn, is implied by the following chain of equalities:

$$
\begin{aligned}
\left\langle T_{k} f, g\right\rangle & =\int_{\hat{\mathbb{R}}^{n}} \hat{f}(\xi) \overline{\hat{g}(\xi) e^{-2 \pi i \xi k}} d \xi \\
& =\sum_{\gamma \in \Gamma^{*}} \int_{\mathbb{T}_{\Gamma^{*}}} \hat{f}(\xi+\gamma) \overline{\hat{g}(\xi+\gamma)} e^{2 \pi i(\xi+\gamma) k} d \xi \\
\text { (since } \gamma k \in \mathbb{Z}) & =\sum_{\gamma \in \Gamma^{*}} \int_{\mathbb{T}_{\Gamma^{*}}} \hat{f}(\xi+\gamma) \overline{\hat{g}(\xi+\gamma)} e^{2 \pi i \xi k} d \xi \\
& =\int_{\mathbb{T}_{\Gamma^{*}}}\left(\sum_{\gamma \in \Gamma^{*}} \hat{f}(\xi+\gamma) \overline{\hat{g}(\xi+\gamma)}\right) e^{2 \pi i \xi k} d \xi \\
& =|\operatorname{det}(c)| \int_{\mathbb{T}_{\Gamma^{*}}}[\hat{f}, \hat{g}]_{\Gamma^{*}}(\xi) e^{2 \pi i \xi k} d \xi .
\end{aligned}
$$


To prove (v) we use the $\Gamma$-periodicity of $M$ and $N$ that ensures

$$
\begin{aligned}
{[M F, N G]_{\Gamma}(x) } & =|\operatorname{det}(c)| \sum_{k \in \Gamma} M(x+k) F(x+k) G^{*}(x+k) N^{*}(x+k) \\
& =|\operatorname{det}(c)| \sum_{k \in \Gamma} M(x) F(x+k) G^{*}(x+k) N^{*}(x) \\
& =M(x)[F, G]_{\Gamma}(x) N^{*}(x) .
\end{aligned}
$$

Corollary 2. Suppose $\Psi$ is a multiwavelet associated with a multigenerated MRA $\left\{V_{j}\right\}_{j \in \mathbb{Z}}$ for $L^{2}\left(\mathbb{R}^{n}\right)$ and $\Phi=\Psi^{0}$ is its multiscaling function. Then, for all $0 \leq$ $\ell, \ell^{\prime} \leq L$,

$$
\left[\hat{\Psi}^{\ell}, \hat{\Psi}^{\ell^{\prime}}\right]_{\Gamma^{*}}(\xi)=|\operatorname{det}(c)|^{-1} \sum_{\gamma \in \Gamma^{*}} \hat{\Psi}^{\ell}(\xi+\gamma) \hat{\Psi}^{\ell^{\prime} *}(\xi+\gamma)=\delta_{\ell, \ell^{\prime}} I_{m+1} \text { for a.e. } \quad \xi
$$

where $\delta_{\ell, \ell^{\prime}}$ is the usual Kronecker delta.

\section{Matricial FiLters}

3.1. Filter conditions. In this section we develop the theory of matricial filters for multigenerated MRA wavelets. Many of the results below are part of the wavelet community folklore; however, we were not able to find a reference where they would be stated at this level of generality. A special case of the theory below can be found, for example, in 11. The general case can be deduced, with some effort and additional (unnecessary) assumptions, from [18. The exposition below contains reasonably transparent new proofs and emphasizes the results that are illustrated in the examples in Section 4

We begin with the following lemma that characterizes functions in $\langle F\rangle_{\Gamma}$.

Lemma 3. Consider $f_{s}, g_{s} \in L^{2}\left(\mathbb{R}^{n}\right), 0 \leq s \leq m$, and let $F$ and $G$ be defined according to (2.3). Assume that $\left\{T_{k} f_{s}: k \in \Gamma, 0 \leq s \leq m\right\}$ is an orthonormal basis for its closed linear span $\langle F\rangle_{\Gamma}$. Then $\langle G\rangle_{\Gamma} \subseteq\langle F\rangle_{\Gamma}$ if and only if there exists a unique $\Gamma^{*}$-periodic matrix $M \in\left(L^{2}\left(\hat{\mathbb{R}}^{n}\right)\right)^{(m+1) \times(m+1)}$ such that

$$
\hat{G}(\xi)=M(\xi) \hat{F}(\xi) \text { for a.e. } \xi \text {. }
$$

Proof. First, we observe that if $M \in\left(L^{2}\left(\hat{\mathbb{R}}^{n}\right)\right)^{(m+1) \times(m+1)}$ is $\Gamma^{*}$-periodic and $\hat{G}(\xi)=M(\xi) \hat{F}(\xi)$ a.e., then $g_{s} \in L^{2}\left(\mathbb{R}^{n}\right), 0 \leq s \leq m$, clearly belong to $\langle F\rangle_{\Gamma}$. Next, we show that if $\langle G\rangle_{\Gamma} \subseteq\langle F\rangle_{\Gamma}$, then

$$
\hat{G}(\xi)=[\hat{G}, \hat{F}]_{\Gamma^{*}}(\xi) \hat{F}(\xi) \text { for a.e. } \xi .
$$

Indeed, by Lemma $1(\mathrm{iv}, \mathrm{v})$,

$$
\left[\hat{G}-[\hat{G}, \hat{F}]_{\Gamma^{*}} \hat{F}, \hat{F}\right]_{\Gamma^{*}}=[\hat{G}, \hat{F}]_{\Gamma^{*}}-[\hat{G}, \hat{F}]_{\Gamma^{*}}[\hat{F}, \hat{F}]_{\Gamma^{*}}=0
$$

Now (3.2) follows from Lemma 1(iii),(iv). Uniqueness of the matrix $M$ with the desired property follows similarly.

Remark 1. Let $F, G$ be as in the above lemma without assuming $\langle G\rangle_{\Gamma} \subseteq\langle F\rangle_{\Gamma}$. Define $H$ via

$$
\hat{H}(\xi)=[\hat{G}, \hat{F}]_{\Gamma^{*}}(\xi) \hat{F}(\xi) \text { for a.e. } \xi \text {. }
$$

Then $\langle H\rangle_{\Gamma}$ is the orthogonal projection of $\langle G\rangle_{\Gamma}$ onto $\langle F\rangle_{\Gamma}$. 
Corollary 4. Suppose $\left\{V_{j}\right\}_{j=-\infty}^{\infty}$ is a multigenerated $M R A$ for $L^{2}\left(\mathbb{R}^{n}\right)$ and $\Phi=$ $\left(\varphi_{0}, \ldots, \varphi_{m}\right)^{T}$ is its scaling function. Then $F \in V_{1}^{m+1}$ if and only if there exists a unique $\Gamma^{*}$-periodic matrix $M_{F} \in\left(L^{2}\left(\hat{\mathbb{R}}^{n}\right)\right)^{(m+1) \times(m+1)}$ such that

$$
\hat{F}(\xi a)=M_{F}(\xi) \hat{\Phi}(\xi) .
$$

The above corollary ensures that the following definition is meaningful.

Definition 7 (Matrix Filters). Let $\left\{V_{j}\right\}_{j=-\infty}^{\infty}$ be a multigenerated MRA for $L^{2}\left(\mathbb{R}^{n}\right)$ and $\Phi=\left(\varphi_{0}, \ldots, \varphi_{m}\right)^{T}$ its scaling function. We define the low pass filter matrix $M_{0}$ via

$$
\hat{\Phi}(\xi a)=M_{0}(\xi) \hat{\Phi}(\xi) .
$$

For $\Psi^{\ell}=\left(\psi_{0}^{\ell}, \ldots, \psi_{m}^{\ell}\right)^{T} \in V_{1}^{m+1}$ we define the high pass filters $M_{\ell}, 1 \leq \ell \leq L$, via

$$
\hat{\Psi}^{\ell}(\xi a)=M_{\ell}(\xi) \hat{\Phi}(\xi) .
$$

We require the following lemma to prove an analog of the Smith-Barnwell equation for matricial filters.

Lemma 5. Let $\left\{\beta_{0}, \ldots, \beta_{L}\right\}$ be a full set of coset representatives of $\Gamma^{*} a^{-1} / \Gamma^{*}$ and $\Phi$ be a multiscaling function of a multigenerated $M R A$ for $L^{2}\left(\mathbb{R}^{n}\right)$. For $j=1,2$, consider $F_{j} \in V_{1}^{m+1}$ such that $\hat{F}_{j}(\xi a)=M_{j}(\xi) \hat{\Phi}(\xi)$. Then

$$
\left[\hat{F}_{1}, \hat{F}_{2}\right]_{\Gamma^{*}}(\xi a)=\sum_{i=0}^{L} M_{1}\left(\xi+\beta_{i}\right) M_{2}^{*}\left(\xi+\beta_{i}\right) \text { for a.e. } \xi .
$$

Proof. To prove (3.6) we use the periodicity of $M_{1}, M_{2}$ and the equalities

$$
\begin{aligned}
& {\left[\hat{F}_{1}, \hat{F}_{2}\right]_{\Gamma^{*}}(\xi a)=|\operatorname{det}(c)|^{-1} \sum_{\gamma \in \Gamma^{*}} \hat{F}_{1}(\xi a+\gamma) \hat{F}_{2}^{*}(\xi a+\gamma)} \\
& =|\operatorname{det}(c)|^{-1} \sum_{\gamma \in \Gamma^{*}} M_{1}\left(\xi+\gamma a^{-1}\right) \Phi\left(\xi+\gamma a^{-1}\right) \Phi^{*}\left(\xi+\gamma a^{-1}\right) M_{2}^{*}\left(\xi+\gamma a^{-1}\right) \\
& =|\operatorname{det}(c)|^{-1} \sum_{i=0}^{L} \sum_{\gamma \in \Gamma^{*}} M_{1}\left(\xi+\beta_{i}\right) \Phi\left(\xi+\beta_{i}+\gamma\right) \Phi^{*}\left(\xi+\beta_{i}+\gamma\right) M_{2}^{*}\left(\xi+\beta_{i}\right) \\
& =\sum_{i=0}^{L} M_{1}\left(\xi+\beta_{i}\right) M_{2}^{*}\left(\xi+\beta_{i}\right) .
\end{aligned}
$$

The last of the above equalities follows from (2.7).

Lemma 6. Let $\left\{\beta_{0}, \ldots, \beta_{L}\right\}$ be a full set of coset representatives of $\Gamma^{*} a^{-1} / \Gamma^{*}$. Let $\Phi=\Psi^{0}$ be the multiscaling function of an $M R A$ for $L^{2}\left(\mathbb{R}^{n}\right)$ with the associated low pass filter matrix $M_{0}(\xi)$. Suppose $\Psi^{\ell} \in V_{1}^{m+1}$ and $M_{\ell}, 1 \leq \ell \leq L$, are the associated high pass filters. Then $\left\{T_{k} \psi_{s}^{\ell}: k \in \Gamma, 0 \leq \ell \leq L, 0 \leq s \leq m\right\}$ is an orthonormal sequence if and only if

$$
\sum_{i=0}^{L} M_{\ell}\left(\xi+\beta_{i}\right) M_{\ell^{\prime}}^{*}\left(\xi+\beta_{i}\right)=\delta_{\ell, \ell^{\prime}} I_{m+1} \text { for } 0 \leq \ell, \ell^{\prime} \leq L .
$$

Proof. Follows from Lemma 5 and Corollary 2 
Definition 8 (Matricial Filter). Let $\left\{V_{j}\right\}_{j=-\infty}^{\infty}$ be an MRA for $L^{2}\left(\mathbb{R}^{n}\right)$ with the associated multiscaling function $\Phi$ and the low pass filter $M_{0}(\xi)$. Also, let $\left\{\beta_{0}, \ldots, \beta_{L}\right\}$ be a full set of coset representatives of $\Gamma^{*} a^{-1} / \Gamma^{*}, \Psi^{\ell} \in V_{1}^{m+1}$, and let $M_{\ell}, 1 \leq \ell \leq L$, be the associated high pass filters. The matricial filter $\mathfrak{M}=\left(\mathfrak{m}_{\ell i}\right)$, $0 \leq \ell, i \leq L$, is the block matrix defined by

$$
\mathfrak{m}_{\ell i}(\xi)=M_{\ell}\left(\xi+\beta_{i}\right) \text {. }
$$

Theorem 7 (Multivariate Unitary Extension Principle). Let $\left\{V_{j}\right\}_{j=-\infty}^{\infty}$ be an MRA for $L^{2}\left(\mathbb{R}^{n}\right)$ with the associated multiscaling function $\Phi$ and the low pass filter $M_{0}(\xi)$. Also, let $\left\{\beta_{0}, \ldots, \beta_{L}\right\}$ be a full set of coset representatives of $\Gamma^{*} a^{-1} / \Gamma^{*}, \Psi^{\ell} \in V_{1}^{m+1}$, and let $M_{\ell}, 1 \leq \ell \leq L$, be the associated high pass filters. Then $\Psi=\left(\psi_{s}^{\ell}\right) \subset L^{2}\left(\mathbb{R}^{n}\right)$, $0 \leq s \leq m, 1 \leq \ell \leq L$, is a multiwavelet for the multigenerated $M R A\left\{V_{j}\right\}_{j=-\infty}^{\infty}$ if and only if the matricial filter $\mathfrak{M}$ is a unitary matrix.

Proof. We need to prove that $\Psi$ is an orthonormal multiwavelet if and only if (3.7) holds. Without loss of generality, assume $\beta_{0}=0$. By Lemma 6, it remains to show only that

$$
V_{1}=\langle\Psi\rangle_{\Gamma}=\left\langle\psi_{s}^{\ell}, 0 \leq \ell \leq L, 0 \leq s \leq m\right\rangle_{\Gamma}
$$

when $\mathfrak{M}$ is unitary. We will use the fact that in this case we have

$$
\sum_{\ell=0}^{L} M_{\ell}^{*}(\xi) M_{\ell}\left(\xi+\beta_{i}\right)=\delta_{i, 0} I_{m+1} \text { for a.e. } \xi \text {. }
$$

Let $F \in V_{1}^{m+1}$. Since $\langle\Psi\rangle_{\Gamma} \subseteq V_{1}$ we only need to show that $F \in\langle\Psi\rangle_{\Gamma}^{m+1}$. By Corollary 4, there exists a $\Gamma^{*}$-periodic matrix filter $M_{F}$ such that $\hat{F}(\xi a)=M_{F}(\xi) \hat{\Phi}(\xi)$. Using (3.8) and (3.5), we get

$$
\begin{aligned}
\hat{F}^{*}(\xi a) & =\hat{\Phi}^{*}(\xi) M_{F}^{*}(\xi) \\
& =\sum_{i=0}^{L} \sum_{\ell=0}^{L} \hat{\Phi}^{*}(\xi) M_{\ell}^{*}(\xi) M_{\ell}\left(\xi+\beta_{i}\right) M_{F}^{*}\left(\xi+\beta_{i}\right) \\
& =\sum_{\ell=0}^{L} \hat{\Psi}_{\ell}^{*}(\xi a) \sum_{i=0}^{L} M_{\ell}\left(\xi+\beta_{i}\right) M_{F}^{*}\left(\xi+\beta_{i}\right)=\sum_{\ell=0}^{L} \hat{\Psi}_{\ell}^{*}(\xi a) N_{F}^{*}(\xi a),
\end{aligned}
$$

where $N_{F}^{*}(\xi)=\sum_{i=0}^{L} M_{\ell}\left(\xi a^{-1}+\beta_{i}\right) M_{F}^{*}\left(\xi a^{-1}+\beta_{i}\right)$ is clearly $\Gamma^{*}$-periodic. Hence, $F \in\langle\Psi\rangle_{\Gamma}^{m+1}$ by Lemma 3

3.2. Composite symmetry. In this subsection we study the symmetry conditions for the filters that would ensure that a multigenerated MRA is, in fact, a composite one.

Definition 9 (Composite Symmetry). A vector function $F \in\left(L^{2}\left(\mathbb{R}^{n}\right)\right)^{m+1}$ has composite symmetry with respect to an $(m+1)$-element group $B$ if (2.1) is satisfied for some ordering of the group $B$.

Clearly, $F$ has composite symmetry if and only if $\hat{F}$ does. Moreover, the normalization condition $a^{-1} B a=B$ ensures that $F$ has composite symmetry if and only if $D_{a} F$ does. Unfortunately, functions with composite symmetry do not form a vector space. Because of this, we fix an ordering of the group $B=\left\{b_{0}=I_{n}, b_{1}, \ldots, b_{m}\right\}$ and, if necessary, reorder composite scaling functions so that they have composite symmetry and (2.1) is satisfied for this particular ordering of the group. 
Assume now that $\Phi$ is a composite multiscaling function and $M_{0}$ is its low pass filter. Since $\Phi$ satisfies (2.1), for each $s=0,1, \ldots, m$ we have

$$
\begin{aligned}
& \sum_{j=0}^{m} m_{s j}(\xi) \hat{\varphi}_{j}(\xi)=\hat{\varphi}_{s}(\xi a)=\hat{\varphi}\left(\xi a b_{s}\right)=\hat{\varphi}\left(\xi b_{\tilde{s}} a\right) \\
& =\sum_{j=0}^{m} m_{0 j}\left(\xi b_{\tilde{s}}\right) \hat{\varphi}_{j}\left(\xi b_{\tilde{s}}\right)=\sum_{j=0}^{m} m_{0 \sigma_{s}(j)}\left(\xi b_{\tilde{s}}\right) \hat{\varphi}_{j}(\xi),
\end{aligned}
$$

where $b_{\tilde{s}}=a b_{s} a^{-1} \in B$ by the normalization condition, and $\sigma_{s} \in S^{m+1}$ is the unique permutation such that $b_{\sigma_{s}(j)}=b_{\tilde{s}}^{-1} b_{j}, 0 \leq j \leq m$. This observation motivates the following definition and proves the subsequent lemma.

Definition 10 (Composite Symmetry of a Matrix). We say that a matrix $M=$ $\left(m_{s j}\right) \in\left(L^{2}\left(\mathbb{T}_{\Gamma}\right)\right)^{(m+1) \times(m+1)}$ has composite symmetry (with respect to the group $B$ and the dilation matrix $a)$ if

$$
m_{s j}(\xi)=m_{0 \sigma_{s}(j)}\left(\xi b_{\tilde{s}}\right), \quad 0 \leq s, j \leq m, \text { for a.e. } \xi
$$

where $b_{\tilde{s}}=a b_{s} a^{-1}$ and $\sigma_{s} \in S^{m+1}$ is the unique permutation such that $b_{\sigma_{s}(j)}=$ $b_{\tilde{s}}^{-1} b_{j}, 0 \leq j \leq m$.

Lemma 8. Let $\left\{V_{j}\right\}_{j \in \mathbb{Z}}$ be an $M R A$ for $L^{2}\left(\mathbb{R}^{n}\right), \Phi=\Psi^{0}$-the associated scaling function, $\Psi$-the associated multiwavelet, and $M_{\ell}, 0 \leq \ell \leq L$-the corresponding matrix filters. Then $\Psi^{\ell}$ has composite symmetry if and only if there exists a permutation matrix $P$ such that $P M_{\ell}$ has composite symmetry, $0 \leq \ell \leq L$.

Remark 2. Equation (3.9) was originally obtained by C. Cabrelli in private communication with U. Molter and the second author.

The following lemmas cover some interesting special cases.

Lemma 9. Assume that the dilation matrix satisfies $|\operatorname{det}(a)|=2$ and $\{0, \beta\}$ is a full set of coset representatives of $\Gamma^{*} a^{-1} / \Gamma^{*}$. Then a filter matrix $M(\xi)$ has composite symmetry if and only if $M(\xi+\beta)$ does.

Proof. Follows from the $\Gamma^{*}$-periodicity of $M$ and the condition $a B a^{-1}=B$.

Lemma 10. Assume that the group $B$ commutes with the dilation matrix a, i.e., $a b=b a$ for all $b \in B$. Then the set of matrices with composite symmetry is an algebra.

Proof. The set of matrices with composite symmetry is always a vector space. To see that it is an algebra when $B$ commutes with $a$, it is enough to notice that in this case $F$ and $D_{a} F$ have composite symmetry with respect to the same ordering of $B$.

3.3. High pass filter constructions. Theorem 7 leaves open two very important questions in constructing multiwavelets from filter matrices. The first asks when it is possible to construct a scaling function given a low pass filter matrix satisfying the Smith-Barnwell equation (3.7) with $\ell=\ell^{\prime}=0$. The second question asks when it is possible to build the matricial filter $\mathfrak{M}$ given its first (block) row. In this paper we limit ourselves to a few special cases when those questions can be answered. We will address the more general situation elsewhere. 
For traditional wavelets with a single scaling function and low pass filter $M_{0}(\xi)$, defined either on $\mathbb{R}$ or by taking Cartesian products of one-dimensional scaling functions for $\mathbb{R}^{n}$, the wavelets are constructed from the high pass filters obtained by translation of the low pass filter by the coset representatives of $\Gamma^{*} a^{-1} / \Gamma^{*}$ and possibly the application of a wavelet multiplier: $M_{\ell}(\xi)=\nu(\xi) M_{0}\left(\xi+\beta_{\ell}\right)$. In general, this construction does not extend to composite dilation wavelets since the wavelet $\psi^{\ell}$ may be defined by multiple scaling functions $\varphi_{s}$ with the same translation $k \in \Gamma$. In this case $\Psi(x)$, defined by $\hat{\Psi}^{\ell}(\xi)=M_{\ell}\left(\xi a^{-1}\right) \hat{\Phi}\left(\xi a^{-1}\right)$, may not be orthogonal to $\hat{\Phi}(x)$.

We conclude this section with three particular methods for completing the matricial filter when $|\operatorname{det}(a)|=2$. Let the coset representatives of $\Gamma^{*} a^{-1} / \Gamma^{*}$ be $\{0, \beta\}$. The following methods apply to three special cases involving $M_{0}(\xi) M_{0}^{*}(\xi+\beta)$.

3.3.1. Filter completion when $M_{0}(\xi) M_{0}^{*}(\xi+\beta)=0$. Suppose $\varphi(x)$ is a Haar-type composite dilation scaling function with $|\operatorname{det}(a)|=2$. Then $M_{0}(\xi)$ satisfies (3.7) and $M_{0}(\xi) M_{0}(\xi+\beta)=0$ for a.e. $\xi$. In this case, the simplest high pass filter construction will succeed. Define $M_{1}(\xi)=M_{0}(\xi+\beta)$. Then

$$
\begin{aligned}
& M_{1}(\xi) M_{1}^{*}(\xi)+M_{1}(\xi+\beta) M_{1}^{*}(\xi+\beta)=M_{0}(\xi+\beta) M_{0}^{*}(\xi+\beta)+M_{0}(\xi) M_{0}^{*}(\xi)=I, \\
& M_{0}(\xi) M_{1}^{*}(\xi)+M_{0}(\xi+\beta) M_{1}^{*}(\xi+\beta)=M_{0}(\xi) M_{0}^{*}(\xi+\beta)+M_{0}(\xi+\beta) M_{0}^{*}(\xi)=0 .
\end{aligned}
$$

Clearly, $M_{0}$ and $M_{1}$ satisfy (3.7) and the matricial filter $\mathfrak{M}$ is unitary. Therefore, $\hat{\Psi}(\xi)=M_{1}\left(\xi a^{-1}\right) \hat{\Phi}\left(\xi a^{-1}\right)$ defines an MRA composite dilation wavelet by Theorem 7 and Lemma 9.

3.3.2. Filter completion when $M_{0}(\xi) M_{0}^{*}(\xi+\beta)$ is self-adjoint. Here we assume that the dilation matrix $a$ satisfies $|\operatorname{det}(a)|=2$ and we have a scaling function $\varphi$ with an associated low pass filter $M_{0}$ such that the matrix $M_{0}(\xi) M_{0}^{*}(\xi+\beta)$ is self-adjoint for a.e. $\xi$. In this case we essentially use the standard [1] construction of the high pass filter modified for the multivariate case [1]. Let $j(a)$ be an index such that $\beta_{j(a)}$ is not an element of the integer lattice and, hence, $e^{2 \pi i \beta_{j(a)}}=-1$. Define $\nu(\xi)=e^{2 \pi i \xi_{j(a)}}$ and let $M_{1}(\xi)=\nu(\xi) M_{0}(\xi+\beta)$. Then

$$
M_{1}(\xi) M_{1}^{*}(\xi)+M_{1}(\xi+\beta) M_{1}^{*}(\xi+\beta)=M_{0}(\xi+\beta) M_{0}^{*}(\xi+\beta)+M_{0}(\xi) M_{0}^{*}(\xi)=I
$$

and

$$
\begin{aligned}
& M_{0}(\xi) M_{1}^{*}(\xi)+M_{0}(\xi+\beta) M_{1}^{*}(\xi+\beta) \\
& \quad=e^{-2 \pi i \xi_{j(a)}}\left(M_{0}(\xi) M_{0}^{*}(\xi+\beta)-M_{0}(\xi+\beta) M_{0}^{*}(\xi)\right)=0,
\end{aligned}
$$

since $M_{0}(\xi) M_{0}^{*}(\xi+\beta)$ is self-adjoint for a.e. $\xi$. Hence, the matricial filter $\mathfrak{M}$ is, indeed, unitary, and $\hat{\Psi}(\xi a)=M_{1}(\xi) \hat{\Phi}(\xi)$ defines an MRA multiwavelet by Theorem 7 This wavelet, however, is likely not to be composite because of the unimodular factor $\nu(\xi)$. Note that all classical wavelets arise from this construction.

3.3.3. Filter completion when $\left(M_{0}(\xi) M_{0}^{*}(\xi+\beta)+M_{0}(\xi+\beta) M_{0}^{*}(\xi)\right)^{2}<I$. Again, we assume that the dilation matrix $a$ satisfies $|\operatorname{det}(a)|=2$ and we have a scaling function $\varphi$ with an associated low pass filter $M_{0}$. Define

$$
D(\xi)=M_{0}(\xi) M_{0}^{*}(\xi+\beta)+M_{0}(\xi+\beta) M_{0}^{*}(\xi),
$$


and assume $\left(I-D^{2}(\xi)\right)>0$ for a.e. $\xi$. In this case, we attempt to find $\left(\Gamma^{*} a^{-1}\right)$ periodic, self-adjoint matrices $A$ and $B$ so that

$$
M_{1}(\xi)=A(\xi) M_{0}(\xi)+B(\xi) M_{0}(\xi+\beta)
$$

will complete a matrix $\mathfrak{M}$ that satisfies the conditions of Theorem 7 . Equivalently, we seek to satisfy (3.7). From the condition $M_{0}(\xi) M_{1}^{*}(\xi)+M_{0}(\xi+\beta) M_{1}^{*}(\xi+\beta)=0$, we get

$$
A(\xi)=-D(\xi) B(\xi) .
$$

It remains only to find a suitable matrix $B(\xi)$. The remaining necessary condition $M_{1}(\xi) M_{1}^{*}(\xi)+M_{1}(\xi+\beta) M_{1}^{*}(\xi+\beta)=I$, provides $B(\xi)\left(I-D^{2}(\xi)\right) B(\xi)=I$. Since $\left(I-D^{2}(\xi)\right)>0$, define

$$
B(\xi)=\left(I-D^{2}(\xi)\right)^{-1 / 2}
$$

By the construction of $A(\xi)$ and $B(\xi)$, the high pass filter matrix $M_{1}(\xi)$ defined by (3.11)-(3.13) satisfies (3.7). Thus, $\mathfrak{M}$ is unitary and $\hat{\Psi}(\xi a)=M_{1}(\xi) \hat{\Phi}(\xi)$ defines an MRA multiwavelet by Theorem 7 . Furthermore, if $B$ commutes with $a$, this multiwavelet will be composite by Lemma 10

We observe that the restriction $\left(I-D^{2}(\xi)\right)>0$ is very often satisfied since this operator is always positive semidefinite. Indeed, we have

$$
I \pm D(\xi)=\left(M_{0}(\xi) \pm M_{0}(\xi+\beta)\right)\left(M_{0}(\xi) \pm M_{0}(\xi+\beta)\right)^{*} .
$$

Clearly, an equivalent restriction is the invertibility of the matrices $\left(M_{0}(\xi) \pm\right.$ $\left.M_{0}(\xi+\beta)\right)$.

\section{EXAMPLES}

4.1. A singly generated Haar-type wavelet in $\mathbb{R}^{3}$. We begin by presenting a singly generated composite Haar-type wavelet in $\mathbb{R}^{3}$. This is the simplest type of example for generating the wavelets as the high pass filter can be constructed from the low pass filter via translation of the low pass filter by a non-zero coset representative of $\Gamma^{*} a^{-1} / \Gamma^{*}$. We present an example in $\mathbb{R}^{3}$, however, this particular wavelet has a straightforward extension to $\mathbb{R}^{n}$.

For this example, let $\Gamma=\mathbb{Z}^{3}$. The composite dilation group, $B$, is the group of reflections through the hyperplanes perpendicular to the standard axes in $\mathbb{R}^{3}$,

$$
b_{0}=\left(\begin{array}{lll}
1 & 0 & 0 \\
0 & 1 & 0 \\
0 & 0 & 1
\end{array}\right), b_{1}=\left(\begin{array}{rrr}
1 & 0 & 0 \\
0 & -1 & 0 \\
0 & 0 & 1
\end{array}\right), b_{2}=\left(\begin{array}{rrr}
1 & 0 & 0 \\
0 & -1 & 0 \\
0 & 0 & -1
\end{array}\right), b_{3}=\left(\begin{array}{rrr}
1 & 0 & 0 \\
0 & 1 & 0 \\
0 & 0 & -1
\end{array}\right),
$$

$b_{4}=-b_{2}, b_{5}=-b_{3}, b_{6}=-b_{0}$, and $b_{7}=-b_{1}$.

The support of the scaling function $\varphi$ is the cube of volume $1 / 8$ in the positive octant, $R=\left\{x: x_{i} \in\left[0, \frac{1}{2}\right]\right\}$. With $\varphi_{0}=\varphi=\frac{1}{\sqrt{8}} \chi_{R_{0}}$ and $R_{0}=R$, we see that the support sets of the functions $\varphi_{s}=D_{b_{s}} \varphi$ are the sets $R_{s}=b_{s} R$. The union of the support sets of the eight scaling functions is the unit cube in $\mathbb{R}^{3}$ centered at the origin; $\bigcup_{s=0}^{7} R_{s}=\left\{x:\left|x_{i}\right| \in\left[0, \frac{1}{2}\right]\right\}$. These support sets are depicted in Figure 1(a).

For the expanding matrix, we take the matrix which doubles the magnitude of the first entry of $x$ and then permutes the entries $x_{i}$ according to $x_{2} \rightarrow x_{1}, x_{3} \rightarrow$ $x_{2}, x_{1} \rightarrow x_{3}$ :

$$
a=\left(\begin{array}{lll}
0 & 1 & 0 \\
0 & 0 & 1 \\
2 & 0 & 0
\end{array}\right)
$$


One interpretation of the action of $a$ on the unit cube $\bigcup_{s=0}^{7} R_{s}$ is a rotation by $\frac{\pi}{2}$ about the $x_{1}$-axis followed by a rotation of $\frac{\pi}{2}$ about the $x_{2}$-axis and an expansion by 2 in the $x_{3}$ direction.

Clearly, $a R_{0}=\left\{x: x_{1}, x_{2} \in\left[0, \frac{1}{2}\right], x_{3} \in[0,1]\right\}$ and therefore $a R_{0}=R_{0} \cup\{x$ : $\left.x_{1}, x_{2} \in\left[0, \frac{1}{2}\right], x_{3} \in\left[\frac{1}{2}, 1\right]\right\}$. From the definition of $R_{3}=b_{3} R_{0}=\left\{x: x_{1}, x_{2} \in\right.$ $\left.\left[0, \frac{1}{2}\right], x_{3} \in\left[-\frac{1}{2}, 0\right]\right\}$, translating $R_{3}$ by the standard basis vector $k_{3}=(0,0,1)^{t}$ will provide the remaining portion of $a R_{0}$. Therefore, $a R_{0}=R_{0} \cup\left(R_{3}+k_{3}\right)$ as shown in Figure 1(b). As a result, our first scaling equation is $\varphi_{0}\left(a^{-1} x\right)=\varphi_{0}(x)+\varphi_{3}\left(x-k_{3}\right)$. The remaining scaling equations are found geometrically following the same basic outline. The scaling equations are:

$$
\begin{array}{ll}
\varphi_{0}\left(a^{-1} x\right)=\varphi_{0}(x)+\varphi_{3}\left(x-k_{3}\right), & \varphi_{4}\left(a^{-1} x\right)=\varphi_{3}(x)+\varphi_{0}\left(x+k_{3}\right), \\
\varphi_{1}\left(a^{-1} x\right)=\varphi_{4}(x)+\varphi_{7}\left(x-k_{3}\right), & \varphi_{5}\left(a^{-1} x\right)=\varphi_{7}(x)+\varphi_{4}\left(x+k_{3}\right), \\
\varphi_{2}\left(a^{-1} x\right)=\varphi_{5}(x)+\varphi_{6}\left(x-k_{3}\right), & \varphi_{6}\left(a^{-1} x\right)=\varphi_{6}(x)+\varphi_{5}\left(x+k_{3}\right), \\
\varphi_{3}\left(a^{-1} x\right)=\varphi_{1}(x)+\varphi_{2}\left(x-k_{3}\right), & \varphi_{7}\left(a^{-1} x\right)=\varphi_{2}(x)+\varphi_{1}\left(x+k_{3}\right) .
\end{array}
$$

After taking the Fourier transform of the scaling equations (4.1), we have:

$$
\begin{array}{rlrl}
\hat{\varphi}_{0}(\xi a) & =\frac{1}{2}\left(\hat{\varphi}_{0}(\xi)+e^{-2 \pi i \xi k_{3}} \hat{\varphi}_{3}(\xi)\right), & \hat{\varphi}_{4}(\xi a)=\frac{1}{2}\left(\hat{\varphi}_{3}(\xi)+e^{2 \pi i \xi k_{3}} \hat{\varphi}_{0}(\xi)\right), \\
\hat{\varphi}_{1}(\xi a)=\frac{1}{2}\left(\hat{\varphi}_{4}(\xi)+e^{-2 \pi i \xi k_{3}} \hat{\varphi}_{7}(\xi)\right), & \hat{\varphi}_{5}(\xi a)=\frac{1}{2}\left(\hat{\varphi}_{7}(\xi)+e^{2 \pi i \xi k_{3}} \hat{\varphi}_{4}(\xi)\right), \\
\hat{\varphi}_{2}(\xi a)=\frac{1}{2}\left(\hat{\varphi}_{5}(\xi)+e^{-2 \pi i \xi k_{3}} \hat{\varphi}_{6}(\xi)\right), & \hat{\varphi}_{6}(\xi a)=\frac{1}{2}\left(\hat{\varphi}_{6}(\xi)+e^{2 \pi i \xi k_{3}} \hat{\varphi}_{5}(\xi)\right), \\
\hat{\varphi}_{3}(\xi a)=\frac{1}{2}\left(\hat{\varphi}_{1}(\xi)+e^{-2 \pi i \xi k_{3}} \hat{\varphi}_{2}(\xi)\right), & \hat{\varphi}_{7}(\xi a)=\frac{1}{2}\left(\hat{\varphi}_{2}(\xi)+e^{2 \pi i \xi k_{3}} \hat{\varphi}_{1}(\xi)\right) .
\end{array}
$$

The low pass filter matrix $M_{0}(\xi)$ is easily extracted from (4.2). Define $(4.3)$

$$
M_{0}(\xi)=\frac{1}{2}\left(\begin{array}{cccccccc}
1 & 0 & 0 & e\left(\xi k_{3}\right) & 0 & 0 & 0 & 0 \\
0 & 0 & 0 & 0 & 1 & 0 & 0 & e\left(\xi k_{3}\right) \\
0 & 0 & 0 & 0 & 0 & 1 & e\left(\xi k_{3}\right) & 0 \\
0 & 1 & e\left(\xi k_{3}\right) & 0 & 0 & 0 & 0 & 0 \\
e\left(-\xi k_{3}\right) & 0 & 0 & 1 & 0 & 0 & 0 & 0 \\
0 & 0 & 0 & 0 & e\left(-\xi k_{3}\right) & 0 & 0 & 1 \\
0 & 0 & 0 & 0 & 0 & e\left(-\xi k_{3}\right) & 1 & 0 \\
0 & e\left(-\xi k_{3}\right) & 1 & 0 & 0 & 0 & 0 & 0
\end{array}\right),
$$

where $e(\alpha):=e^{-2 \pi i \alpha}$.

Thus, we have the desired Fourier transform version of the scaling equation,

$$
\hat{\Phi}(\xi a)=M_{0}(\xi) \hat{\Phi}(\xi) .
$$

Since $|\operatorname{det}(a)|=2$, there are two cosets of the quotient $\Gamma^{*} a^{-1} / \Gamma^{*}$, namely $\{0, \beta\}$ with $\beta=(0,0,1 / 2)$. From (4.3), we have $M_{0}(\xi) M_{0}^{*}(\xi+\beta)=0$. Hence, we define $M_{1}(\xi)=M_{0}(\xi+\beta)$ as in Section 3.3 .1 to see that the vector-valued function satisfying

$$
\hat{\Psi}(\xi a)=M_{1}(\xi) \hat{\Psi}(\xi)
$$

defines a Haar-type, MRA, composite dilation wavelet. Expanding (4.5), taking the inverse Fourier transform, and extracting the first row provides

$$
\psi(x)=\varphi_{0}(a x)-\varphi_{3}\left(a x-k_{3}\right) .
$$

Therefore, $\psi$ is a singly generated, Haar-type composite dilation wavelet for $\mathbb{R}^{3}$. 


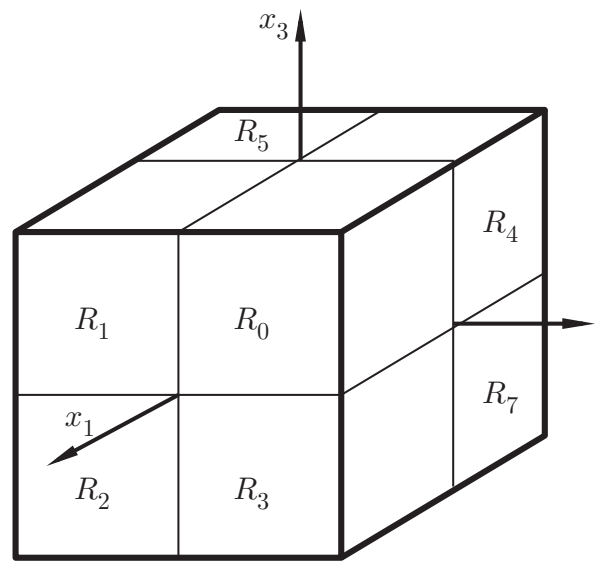

(a)

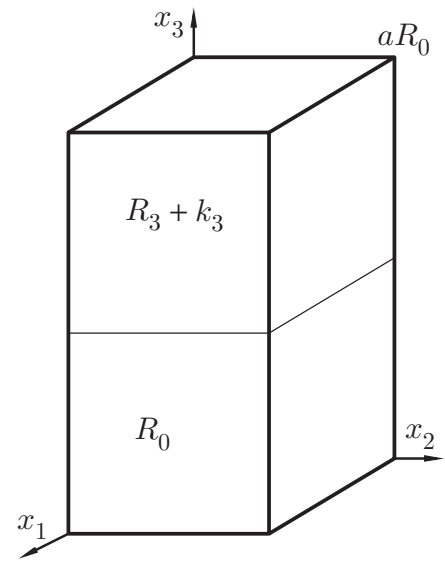

(b)

Figure 1. (a) The support sets $R_{s}, s=0, \ldots, 7$ for the scaling functions $\varphi_{s}$ defined in (4.1). (b) The support sets of the dilated composite scaling function $\varphi\left(a^{-1} x\right)$ and composite dilation wavelet $\psi\left(a^{-1} x\right)$ defined in (4.1) and (4.6).

4.2. A Haar-type composite dilation wavelet for $\mathbb{R}^{2}$. In this section, we apply the theory of Section 3 to a known Haar-type wavelet for $\mathbb{R}^{2}$. Krishtal et al. [13] provided the existence of Haar-type composite dilation wavelets in $\mathbb{R}^{2}$ by presenting three examples. In their discussion, they presented filter equations when $B$ was the group of full symmetries of the square. The other two examples involve the hexagonal lattice and subsets of the hexagon as support sets for the scaling function. Here we present the matricial filter equations for the group of full symmetries of the hexagon and show that these filters satisfy the necessary and sufficient condition (3.7). Blanchard and Steffen [3] have cataloged the Haar-type composite dilation wavelets when the semi-direct product $B \ltimes \Gamma$ forms a crystallographic group on $\mathbb{R}^{2}$ (also called a plane symmetry group). Independently, MacArthur and Taylor have utilized the representation theoretic framework to produce Haar-type wavelets from the plane crystallographic groups [17] and MacArthur also cataloged these wavelets in $\mathbb{R}^{2}[16]$.

Let $B$ be the group of full symmetries of the hexagon and $\Gamma=c \mathbb{Z}^{2}$ where $c=\frac{1}{4}\left(\begin{array}{cc}3 \sqrt{3} & 0 \\ 3 & 6\end{array}\right)$. (In the plane symmetry (crystallographic) group notation from [3], $B \ltimes \Gamma=\mathrm{p} 6 \mathrm{~m}$.) Choose the ordering of $B$ to map the set $R_{0}$ counterclockwise around the octagon (see Figure 2(a)). The columns of the matrix $c$ provide two generating vectors for the full rank lattice $\Gamma$. The dual lattice $\Gamma^{*}$ is generated by $\left(\frac{4 \sqrt{3}}{9}, 0\right)$ and $\left(\frac{-2 \sqrt{3}}{9}, \frac{2}{3}\right)$. Finally, the composite dilation system is completed by the dilation matrix $a=\frac{1}{2}\left(\begin{array}{cc}3 & -\sqrt{3} \\ \sqrt{3} & 3\end{array}\right)$.

Let $k_{0}=\left(\frac{3 \sqrt{3}}{4}, \frac{3}{4}\right)^{t}$ and $k_{1}=\left(0, \frac{3}{2}\right)^{t}$, namely the columns of the matrix $c$ which form a basis for the lattice $\Gamma$. For brevity, let $k_{01}=k_{0}-k_{1}$. Then we define the 


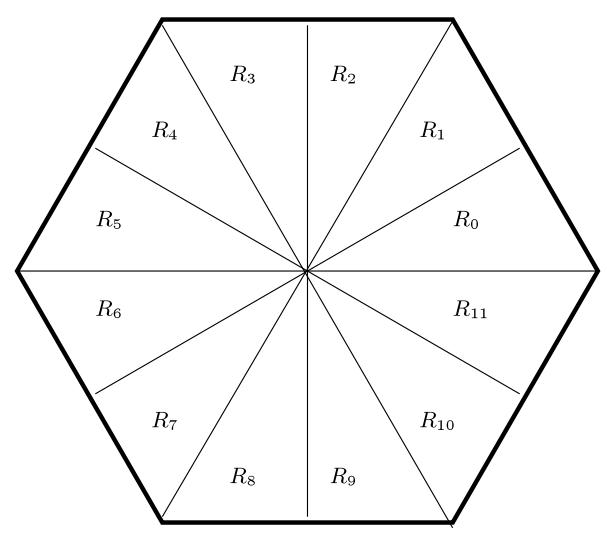

(a)

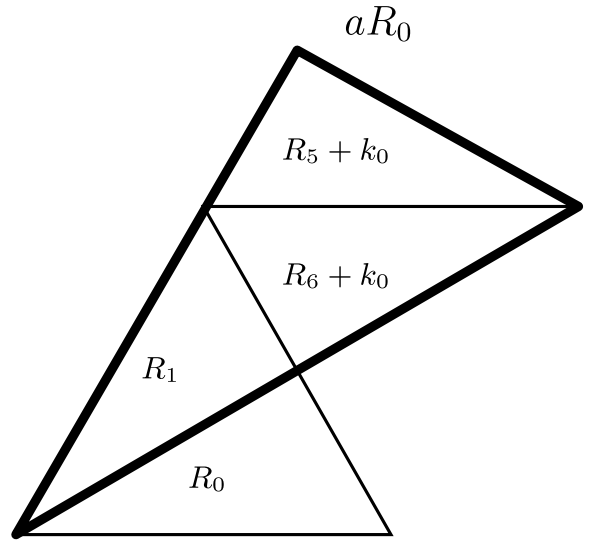

(b)

Figure 2. (a) The support sets $R_{s}, s=0, \ldots, 11$ for the scaling functions $\varphi_{s}$ defined in (4.8). (b) The support sets of the dilated composite scaling function $\varphi\left(a^{-1} x\right)$ and composite dilation wavelet $\psi\left(a^{-1} x\right)$ defined in (4.8) and (4.9).

low pass filter

$$
M_{0}(\xi)=\frac{1}{3}\left(\begin{array}{cc}
0 & 1 \\
0 & 0 \\
0 & 0 \\
e\left(-\xi k_{01}\right) & 0 \\
0 & 0 \\
0 & e\left(-\xi k_{0}\right) \\
e\left(-\xi k_{0}\right) & 0 \\
0 & 0 \\
0 & e\left(-\xi k_{1}\right) \\
0 & 0 \\
0 & 0 \\
1 & 0
\end{array}\right.
$$
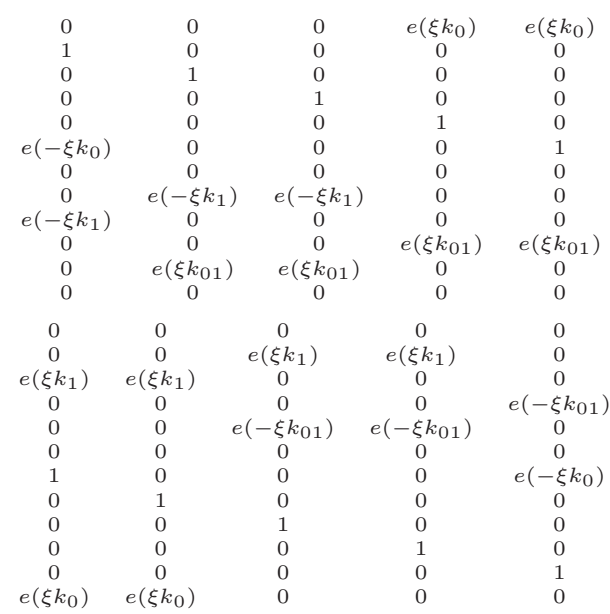

$$
\begin{gathered}
0 \\
0 \\
e\left(\xi k_{1}\right) \\
0 \\
0 \\
0 \\
0 \\
1 \\
0 \\
0 \\
0 \\
e\left(\xi k_{0}\right)
\end{gathered}
$$$$
\begin{gathered}
0 \\
e\left(\xi k_{1}\right) \\
0 \\
0 \\
e\left(-\xi k_{01}\right) \\
0 \\
0 \\
0 \\
1 \\
0 \\
0 \\
0
\end{gathered}
$$

$$
\left.\begin{array}{cc}
0 & 0 \\
e\left(\xi k_{1}\right) & 0 \\
0 & 0 \\
0 & e\left(-\xi k_{01}\right) \\
e\left(-\xi k_{01}\right) & 0 \\
0 & 0 \\
0 & e\left(-\xi k_{0}\right) \\
0 & 0 \\
0 & 0 \\
1 & 0 \\
0 & 1 \\
0 & 0
\end{array}\right)
$$

Then $\hat{\Phi}(\xi a)=M_{0}(\xi) \hat{\Phi}(\xi)$ and the inverse Fourier transform of the first row of this vector equation yields the obvious scaling equation for the scaling function $\varphi(x)=\frac{4 \sqrt{2 \sqrt{3}}}{3} \chi_{R}$ where $R=R_{0}$ is the fundamental triangle for the Hexagon as shown in Figure 2. This scaling equation, with $\varphi:=\varphi_{0}$ is

$$
\varphi_{0}\left(a^{-1} x\right)=\varphi_{1}(x)+\varphi_{5}\left(x-k_{0}\right)+\varphi_{6}\left(x-k_{0}\right) .
$$

From (4.8), the dilated scaling function $\varphi:=\varphi_{0}$ is, as expected, the sum of three translated scaling functions. However, two of these scaling functions require an identical translation, namely a translation by $k_{0}$. Note that $\operatorname{det}(a)=3$ so the constructions from Section 3.3 do not apply. Instead, Krishtal et al. 13] determined 
the orthogonal wavelets geometrically. Following [13, two wavelet generators for the composite dilation system are defined by

$$
\begin{aligned}
& \psi^{1}\left(a^{-1} x\right)=\frac{1}{\sqrt{6}}\left(\varphi_{1}(x)-\varphi_{6}\left(x-k_{0}\right)\right), \\
& \psi^{2}\left(a^{-1} x\right)=\frac{1}{3 \sqrt{2}}\left(\varphi_{1}(x)-2 \varphi_{5}\left(x-k_{0}\right)+\varphi_{6}\left(x-k_{0}\right)\right) .
\end{aligned}
$$

By taking the Fourier transforms of (4.9) and each of the dilations by elements of the group $B$, we determine the two highpass filters:

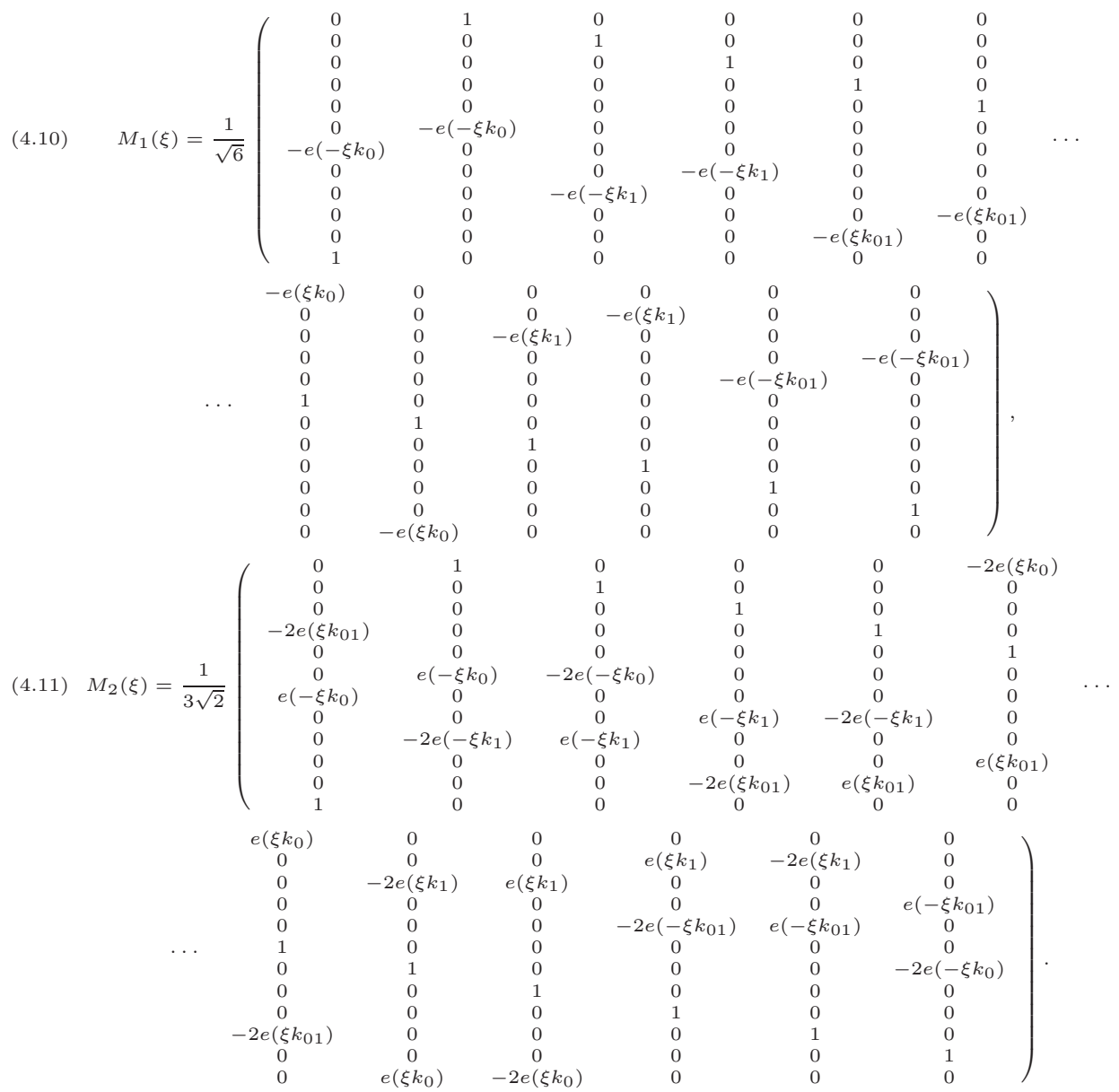

To check the filter conditions (3.7) for these $12 \times 12$ matrices is clearly tedious. However, the symbolic toolbox in MatLab allows us to do so easily (with software available on the first author's web page). The matrix filters $M_{0}(\xi), M_{1}(\xi), M_{2}(\xi)$ do indeed produce a unitary matricial filter $\mathfrak{M}$, and, by Theorem 7 and Lemma 8 define a Haar-type, MRA, composite dilation wavelet system for $L^{2}\left(\mathbb{R}^{2}\right)$.

4.3. A composite dilation wavelet for $L^{2}(\mathbb{R})$ with accuracy 2. In previous work appearing in [2, the current authors found the first known compactly supported composite dilation wavelet with accuracy greater than the Haar-type 
wavelets. Recall that a function $f(x)$ has accuracy $p$ if, for every $q<p, q, p \in \mathbb{N}$, the restriction of each monomial $x^{q}$ to a compact set is a linear combination of the $\Gamma$-translations of $f$; i.e., $x^{q} \chi_{K}(x)=\sum_{k \in \Gamma} \alpha_{k} f(x-k) \chi_{K}(x)$, where $K \subset \mathbb{R}$ is compact and $\left\{\alpha_{k}\right\}$ is a sequence of complex coefficients. In this section, we describe this composite dilation wavelet with accuracy 2 which generates an orthonormal basis for $L^{2}(\mathbb{R})$. This compactly supported, piecewise-linear CDW was developed in 2 by first finding a scaling function with accuracy 2 via the accuracy equations for refinable functions determined by Cabrelli, Heil, and Molter 4.

The composite structure provides relations between the coefficients in the refinement equations from [4] which lead to a system of equations whose solution will determine a scaling function for $\mathbb{R}$. In $\mathbb{R}$, the only finite group $B$ is the reflection through the origin, namely $B=\{1,-1\}$. The integer lattice and the dyadic dilation $(a=2)$ define the remainder of the composite dilation system.

By solving the (rather large) system of quadratic equations produced by the accuracy equations for accuracy 2 , a scaling function for a composite dilation MRA on $\mathbb{R}$ was found to be

$$
\varphi(x)= \begin{cases}\frac{1}{\sqrt{2}}[2 \sqrt{3} x+(1-\sqrt{3})] & \text { for } x \in[0,1], \\ 0 & \text { for } x \notin[0,1]\end{cases}
$$

almost everywhere.

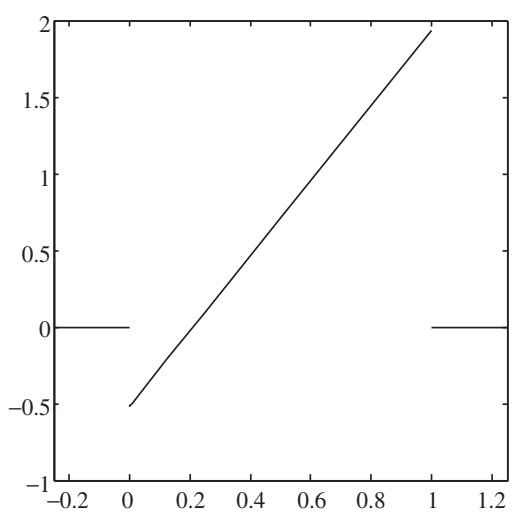

(a)

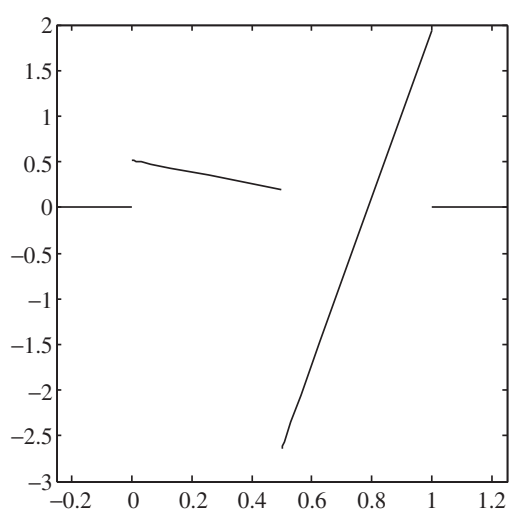

(b)

Figure 3. (a) The composite dilation scaling function $\varphi$ defined in (4.12). (b) The composite dilation wavelet $\psi$ defined in (4.21).

From (4.12), we apply the dilations by the composite dilation group $B$ and define the functions $\varphi_{0}(x)=D_{1} \varphi(x)=\varphi(x)$ and $\varphi_{1}(x)=D_{-1} \varphi(x)=\varphi(-x)$. The solution of the refinement equations determined the following scaling (refinement) equations:

$$
\begin{aligned}
& \varphi_{0}\left(\frac{1}{2} x\right)=\alpha \varphi_{0}(x)+\beta \varphi_{0}(x-1)+\gamma \varphi_{1}(x-1)+\delta \varphi_{1}(x-2), \\
& \varphi_{1}\left(\frac{1}{2} x\right)=\alpha \varphi_{1}(x)+\beta \varphi_{1}(x+1)+\gamma \varphi_{0}(x+1)+\delta \varphi_{0}(x+2),
\end{aligned}
$$


where $\alpha=\frac{3-\sqrt{3}}{4}, \beta=\frac{3+\sqrt{3}}{4}, \gamma=\frac{1-\sqrt{3}}{4}$, and $\delta=\frac{1+\sqrt{3}}{4}$. Taking the Fourier transform of (4.13), we construct the low pass filter matrix

$$
M_{0}(\xi)=\frac{1}{2}\left(\begin{array}{cc}
\alpha+\beta e^{-2 \pi i \xi} & \gamma e^{-2 \pi i \xi}+\delta e^{-4 \pi i \xi} \\
\gamma e^{2 \pi i \xi}+\delta e^{4 \pi i \xi} & \alpha+\beta e^{2 \pi i \xi}
\end{array}\right) .
$$

Then we have the vector-valued scaling equation $\hat{\Phi}(\xi a)=M_{0}(\xi) \hat{\Phi}(\xi)$ as desired. A simple calculation shows that $\alpha^{2}+\beta^{2}+\gamma^{2}+\delta^{2}=2$ and $\alpha \delta+\beta \gamma=0$, and therefore,

$$
\begin{aligned}
M_{0}(\xi) M_{0}^{*}(\xi)+M_{0}\left(\xi+\frac{1}{2}\right) & M_{0}^{*}\left(\xi+\frac{1}{2}\right) \\
& =\frac{1}{2}\left(\begin{array}{cc}
\alpha^{2}+\beta^{2}+\gamma^{2}+\delta^{2} & 2(\alpha \delta+\beta \gamma) e^{-4 \pi i \xi} \\
2(\alpha \delta+\beta \gamma) e^{4 \pi i \xi} & \alpha^{2}+\beta^{2}+\gamma^{2}+\delta^{2}
\end{array}\right) \\
& =\left(\begin{array}{cc}
1 & 0 \\
0 & 1
\end{array}\right) .
\end{aligned}
$$

If we let $N(\xi)=M_{0}(\xi+1 / 2)$, the translation of the low pass filter by the non-zero coset representative, (4.15) shows that $N(\xi)$ satisfies (3.7) with $\ell=\ell^{\prime}=1$. However, the function defined by $\hat{F}(x)=N(\xi) \hat{\Phi}(\xi)$ is not orthogonal to the scaling function $\Phi(x)$. Therefore, we must use an alternative method to determine the wavelet, and in this case, we employ the high pass filter construction from Section 3.3.3. Let

$$
\begin{aligned}
D(\xi) & =M_{0}(\xi) M_{0}^{*}\left(\xi+\frac{1}{2}\right)+M_{0}\left(\xi+\frac{1}{2}\right) M_{0}^{*}(\xi) \\
& =\frac{1}{2}\left(\begin{array}{cc}
\alpha^{2}-\beta^{2}-\gamma^{2}+\delta^{2} & 2(\alpha \delta-\beta \gamma) e^{-4 \pi i \xi} \\
2(\alpha \delta-\beta \gamma) e^{-4 \pi i \xi} & \alpha^{2}-\beta^{2}-\gamma^{2}+\delta^{2}
\end{array}\right) \\
& =\frac{\sqrt{3}}{4}\left(\begin{array}{cc}
-1 & e^{-4 \pi i \xi} \\
e^{4 \pi i \xi} & -1
\end{array}\right) .
\end{aligned}
$$

Then $\left(I-D^{2}(\xi)\right)>0$ and this method for completing the matricial filter $\mathfrak{M}$ applies.

Following Section 3.3.3. let $B(\xi)=\left(I-D^{2}(\xi)\right)^{-1 / 2}$. Then, one choice for the square root is

$$
B(\xi)=\frac{1}{2}\left(\begin{array}{cc}
-3 & e^{-4 \pi i \xi} \\
e^{4 \pi i \xi} & -3
\end{array}\right)
$$

Now we define $A(\xi)=-B(\xi) D(\xi)$ so that

$$
A(\xi)=\frac{\sqrt{3}}{2}\left(\begin{array}{cc}
-1 & e^{-4 \pi i \xi} \\
e^{4 \pi i \xi} & -1
\end{array}\right) .
$$

From (4.17) and (4.18), we define the high pass filter matrix as $M_{1}(\xi)=A(\xi) M_{0}(\xi)+$ $B(\xi) M_{0}(\xi+1 / 2)$. A series of straightforward computations leads to

$$
M_{1}(\xi)=\frac{1}{2}\left(\begin{array}{cc}
-\gamma+\delta e^{-2 \pi i \xi} & \alpha e^{-2 \pi i \xi}-\beta e^{-4 \pi i \xi} \\
\alpha e^{2 \pi i \xi}-\beta e^{4 \pi i \xi} & -\gamma+\delta e^{2 \pi i \xi}
\end{array}\right) .
$$

From Section 3.3.3, we know that $M_{1}(\xi)$ is a high pass filter. It is interesting to note that

$$
\begin{aligned}
M_{1}(\xi) M_{1}^{*}(\xi)+M_{1}\left(\xi+\frac{1}{2}\right) & M_{1}^{*}\left(\xi+\frac{1}{2}\right) \\
& =\frac{1}{2}\left(\begin{array}{cc}
\alpha^{2}+\beta^{2}+\gamma^{2}+\delta^{2} & 2(\alpha \delta+\beta \gamma) e^{-4 \pi i \xi} \\
2(\alpha \delta+\beta \gamma) e^{4 \pi i \xi} & \alpha^{2}+\beta^{2}+\gamma^{2}+\delta^{2}
\end{array}\right),
\end{aligned}
$$


which is the identity by (4.15). As shown in Section 3.3.3. $\hat{\Psi}(\xi)=M_{1}(\xi / 2) \hat{\Phi}(\xi / 2)$ defines an MRA, composite dilation wavelet.

Taking the inverse Fourier transform of $\hat{\Psi}(\xi)=M_{1}(\xi / 2) \hat{\Phi}(\xi / 2)$ and extracting the first row, the wavelet is defined by

$$
\psi(x)=-\gamma \varphi_{0}(2 x)+\delta \varphi_{0}(2 x-1)+\alpha \varphi_{1}(2 x-1)-\beta \varphi_{1}(2 x-2) .
$$

Another straightforward calculation shows that the composite dilation wavelet, displayed in Figure 3(b), is, almost everywhere, the piecewise linear function

$$
\psi(x)= \begin{cases}\frac{1}{\sqrt{2}}[(6-4 \sqrt{3}) x-(1-\sqrt{3})] & \text { for } x \in\left[0, \frac{1}{2}\right), \\ \frac{1}{\sqrt{2}}[(6+4 \sqrt{3}) x-(5+3 \sqrt{3})] & \text { for } x \in\left[\frac{1}{2}, 1\right], \\ 0 & \text { for } x \notin[0,1] .\end{cases}
$$

In 2, the composite dilation wavelet $\psi$ was determined via brute force. The filter equations and constructions from Section 3 are a considerable improvement that we hope will lead to the discovery of smoother compactly supported composite dilation wavelets.

\section{ACKNOWLEDGMENTS}

The authors wish to thank Guido Weiss and Ed Wilson for their guidance and mentorship, and for introducing us to composite dilation wavelets.

\section{REFERENCES}

[1] D. Bakić, I. Krishtal, and E. N. Wilson. Parseval frame wavelets with $E_{n}^{(2)}$-dilations. Appl. Comput. Harmon. Anal., 19(3):386-431, 2005. MR2186451 (2006g:42056)

[2] J. D. Blanchard. Existence and accuracy results for composite dilation wavelets. Ph.D. dissertation, 2007. Washington University in St. Louis. MR2710862

[3] J. D. Blanchard and K. R. Steffen. Crystallographic Haar-type composite dilation wavelets. In Wavelets and Multi-scale Analysis: theory and applications. Birkhäuser Boston, Inc., March 2011.

[4] C. Cabrelli, C. Heil, and U. Molter. Accuracy of lattice translates of several multidimensional refinable functions. J. Approx. Theory, 95(1):5-52, 1998. MR.1645975 (99g:42038)

[5] F. Colonna, G. Easley, K. Guo, and D. Labate. Radon transform inversion using the shearlet representation. Appl. Comput. Harmon. Anal., 29(2):232-250, 2010. MR2652460

[6] K. Guo, G. Kutyniok, and D. Labate. Sparse multidimensional representations using anisotropic dilation and shear operators. In Wavelets and splines: Athens 2005, Mod. Methods Math., pages 189-201. Nashboro Press, Brentwood, TN, 2006. MR2233452 (2007c:42050)

[7] K. Guo and D. Labate. Optimally sparse multidimensional representation using shearlets. SIAM J. Math. Anal., 39(1):298-318, 2007. MR2318387 (2008k:42097)

[8] K. Guo and D. Labate. Characterization and analysis of edges using the continuous shearlet transform. SIAM J. Imaging Sci., 2(3):959-986, 2009. MR.2551249 (2011b:94004)

[9] K. Guo, D. Labate, W. Lim, G. Weiss, and E. N. Wilson. The theory of wavelets with composite dilations. In Harmonic analysis and applications, Appl. Numer. Harmon. Anal., pages 231-250. Birkhäuser Boston, Boston, MA, 2006. MR 2249312 (2007d:42072)

[10] K. Guo, D. Labate, W. Lim, G. Weiss, and E. N. Wilson. Wavelets with composite dilations and their MRA properties. Appl. Comput. Harmon. Anal., 20(2):202-236, 2006. MR 2207836 (2006j:42056)

[11] E. Hernández and G. Weiss. A first course on wavelets. Studies in Advanced Mathematics. CRC Press, Boca Raton, FL, 1996. MR,1408902 (97i:42015)

[12] R. Houska. The nonexistence of shearlet-like scaling multifunctions that satisfy certain minimally desirable properties and characterizations of the reproducing properties of the integer lattice translations of a countable collection of square integrable functions. Ph.D. dissertation, 2009. Washington University in St. Louis. MR2713543 
[13] I. Krishtal, B. Robinson, G. Weiss, and E. N. Wilson. Some simple Haar-type wavelets in higher dimensions. J. Geom. Anal., 17(1):87-96, 2007. MR22302875 (2008a:42018)

[14] G. Kutyniok, J. Lemvig, and W.-Q Lim. Compactly supported shearlets. submitted, 2010.

[15] W.-Q Lim. The discrete shearlet transform: A new directional transform and compactly supported shearlet frames. IEEE Trans. Image Processing, 19(5):1166-1180, 2010. MR 2723739

[16] J. MacArthur. Compatible dilations and wavelets for the wallpaper groups. preprint, 2009.

[17] J. MacArthur and K. Taylor. Wavelets with crystal symmetry shifts. submitted, 2009.

[18] A. Ron and Z. Shen. Affine systems in $L_{2}\left(\mathbb{R}^{d}\right)$ : the analysis of the analysis operator. J. Funct. Anal., 148(2):408-447, 1997. MR1469348 (99g:42043)

Department of Mathematics and Statistics, Grinnell College, Grinnell, Iowa 50112

E-mail address: jeff@math.grinnell.edu

$U R L:$ http://www.math.grinnell.edu/ blanchaj

Department of Mathematics, Northern Illinois University, Dekalb, Illinois 60115

E-mail address: krishtal@niu.edu

URL: http://www.math.niu.edu/ krishtal 